\title{
Epidemiological profile of the imported Malaria in the north region of Morocco from 2014 to 2018
}

\author{
Kaoutar Nabah ${ }^{1 *}$, Nadya Mezzoug ${ }^{2}$, Ahmed Aarab ${ }^{3}$,Halima Oufdou ${ }^{4}$, and Kacem Rharrabe ${ }^{1}$ \\ ${ }^{1}$ Abdelmalek Essaadi University. Polydisciplinary Faculty - Larache. Morocco. \\ ${ }^{2}$ Abdelmalek Essaadi University. Faculty of Sciences-Tetouan. Morocco. \\ ${ }^{3}$ Abdelmalek Essaadi University. Faculty of Sciences and technology -Tangier. Morocco. \\ ${ }^{4}$ Mohammed V University - Rabat. Morocco
}

\begin{abstract}
In Morocco, the epidemiological surveillance of imported Malaria still be the pillar of the eradication strategy of the disease as part of environmental health. The aim of this study is to describe the epidemiological profile of malaria in north region of Morocco. It is retrospective descriptive study, based on program reports, carried out from 28 April 2019 to 18 February 2020 covering 2014-2018 period. The statistical analysis is performed by Epiinfo 7. In total, 59 cases of imported Malaria were reported. TangierAssilah province presented the majority of the cases (62.7\%). The frequency was highest in 2018 with $35,2 \%$ (19) of cases. The male patient (79.7\%) and the age of] $15-30]$ years (50\%) were the most detected. 55.5\% of the cases had a Moroccan nationality and were travelling from Guinea (33.9\%). Diagnosis was conducting less than 2 days in $75 \%$ since the onset of clinical signs. The parasite was Plasmodium falciparum for $84.7 \%$ of Malaria cases. In conclusion, to prevent the risk of re-emergence of the disease in the region, the prevention and control measures against the imported malaria can be established at the regional as well as national level to achieve the objective of the malaria eradication in Morocco.
\end{abstract}

\section{Introduction}

Globally, the malaria control has prevented 1.5 billion cases and 7.6 million deaths since 1990[1]. In 2018, WHO reported 228 million malaria cases, compared to 251 million in 2010 [1]. The number of countries that were malaria endemic in 2000 and that reported fewer than 10 000 malaria cases increased from 26 in 2000 to 46 in $2019[1]$.

In the same period, the number of countries with fewer than 100 indigenous cases increased from six to 27[1]. The WHO African region alone recorded $93 \%$ of malaria cases and $94 \%$ of malaria-related deaths worldwide in 2018 [1].

A Global Technical Strategy for Malaria 2016-2030 was adopted by the World Health Assembly in May 2015 [2]. The strategy aims to reduce malaria mortality rates and case incidence by at least $90 \%$ by 2030 worldwide [2] and also to prevent a re-emergence of malaria in all countries that are malaria-free including Morocco[2]. Between 2000 and 2019, no country that was certified malaria free has been found to have malaria transmission re-established.

However in the context of Corona virus disease (COVID-19) pandemic during 2020; the progress report of the malaria control strategy reported a delay on the decrease of the mortality and incidence rate $(18 \%$ and $3 \%$ successively) compared to what was traced in $2020(40 \%$ for both) [3]. Thus the improvement of the malaria measures control is required worldwide.

In Morocco, no case of indigenous malaria has been recorded since 2005[4]. This allowed it to have certification of malaria-free country in 2010 after accomplishing the procedures and standards required by
WHO [5]. To keep this situation stable, the surveillance of the imported malaria cases is a big issue to prevent the new introduction of the malaria into the country.

In 2017, the Moroccan health ministry notified 291 of the imported malaria cases [6]. The average of those imported cases between 2012 and 2016 was 255.60 cases per year [7]. The majority of the cases are adults and males coming from endemic areas specially the African countries [8].

The Plasmodium falciparum parasite was the responsible of the infection of most cases imported into Morocco [8]. The detection and early treatment of imported malaria cases as well as the upkeep of the vector control are the leading axes of the prevention strategy of the reintroduction of the malaria in the country [9].

Tangier Tetouan Al-Hoceima (TTA) is a region in northern of Morocco with a population of 3,903,744 [10]. The region contributes by $25.2 \%$ of the national GDP and having significant economic development with a growth rate of $7.6 \%$ in 2018 [11]. The north region, as growing commercial and economic pole, continues to perform an increase of the foreign commercial exchanges worldwide, mainly with the African areas. Thereafter, the surveillance of the imported malaria cases is needed.

The objective of our study is to analyse the epidemiological profile of imported malaria cases in the north region of Morocco during the period 2014-2018, to improve the epidemiological surveillance of this disease.

\footnotetext{
*Corresponding author: kaoutaysf@gmail.com
} 
Table 1: The epidemiological profile of malaria cases imported to the north region of Morocco; 2014-2018

Province (n=59)
Fahse-Anjra
Al-Hoceima
Larache
M'diq-Fnideq
Tangier-Assilah
Tetouan

Frequency $\quad$ Percent $\%$

Year of notification $(\mathbf{n}=59)$

2014

2015

2016

2017

2018

Age range (years) $(n=58)$

[0-15]

]15-30]

]30-45]

$>45$

\section{Gender $(\mathbf{n}=56)$}

Female

Male

Profession $(\mathbf{n}=7)$

Driver

Student

Employer in marine

Worker

Country of last travelling $(n=54)$

Ivory coast

Guinea

Mali

Philippine

Others*

Nationality $(\mathbf{n}=9)$

Guinea

Morocco

Niger

Philippine

Chad

Parasite $(\mathbf{n}=\mathbf{5 9})$

Plasmodium falciparum

Plasmodium malaria

Plasmodium ovale
813,6

$1 \quad 1,7$

$1 \quad 1,7$

$5 \quad 8,5$

$37 \quad 62,7$

$7 \quad 11,8$

$8 \quad 13,6$

1322,0

$5 \quad 8,5$

$14 \quad 23,7$

$19 \quad 32,2$

610,4

$29 \quad 50,0$

$17 \quad 29,3$

$6 \quad 10,3$

$\begin{array}{ll}47 & 79,7\end{array}$

$1 \quad 14,2$

228,6

228,6

228,6

$14 \quad 25,9$

$20 \quad 37,0$

59,3

$5 \quad 9,3$

1018,5

$5 \quad 55,6$

111,1

$1 \quad 11,1$

111,1

$50 \quad 84,7$

$1 \quad 1,7$

$8 \quad 13,6$ 


\section{Materials and methods}

This is a retrospective descriptive epidemiological study with a quantitative approach carried out between $04 / 28 / 2019$ and $02 / 18 / 2020$ in Tangier Tetouan AlHoceima region. The data of 2014-2018 years are collected from the annual reports of the malaria control program at primary health care network of the Health Delegations of the region provinces.

The distribution by province, year of study, age, gender, gender by age, the country of the last displacement of the case and the parasite responsible of the infection were the main variables studied of imported malaria case. Also, the frequency of those variables during the study period has been calculated. The diagnostic delay, the treatment delay and the parasite identification delay were identified.

The database was cleaned using Excel. Statistical analysis univariate and bivariat is performed by EpiInfo 7. Zotero (reference management) and NVIVO (contextual analysis of articles) software are used for bibliographic research on the study subject.

\section{Results}

In our study, 59 cases of imported malaria were reported between 2014 and 2018 in the Tangier Tetouan Al-Hoceima region (Table 1). In the province of TangierAssilah, the percentage of the number of cases was $62.7 \%$ (37). In the province of Fahse-Anjra, 13.6\% (8) of cases were recorded and $11.8 \%$ (7) recorded in Tetouan. Only one case was in Larache and Al-Hoceima provinces, representing each one $1.7 \%$ (1) of the total cases.

Between 2014 and 2018, it was an increase in the number of cases during these years of study: $8(6 \%)$ in 2014 to $19(32.3 \%)$ cases in 2018 .

The median age of imported malaria to the region was 28.5 [23-35] years old with a minimum of 13 months and a maximum of 65 years old. The mode was at 24 years old.The frequency of $50 \%$ (29) of cases were ]15-30] years old, followed by $29.3 \%$ (17) of cases agedby] 30 45] years old. Children with [0 -15] years old and adults older than 45 years old were $10.4 \%$ (6) of the cases each.

The majority of patients were male representing $79.7 \%$ (47) of cases against only $20.3 \%$ (12) of women. Only 7 cases reported their professions, 2 of them were students.

The association between the gender and age ranged between $\leq 18$ years and $>18$ years was statistically significant $(p=0,03)$ by Fisher Exacte test (table 2). Thereafter, the men with the age superior to 18 years olds were significantly the most affected group by malaria disease.

Imported malaria cases have been moved to Guinea (37\%) and Ivory Coast (25.9\%). The percentage of 55,6\% of imported malaria cases had Moroccan nationality. (Table 1)

The identification of the parasite was in $84.7 \%$ of cases Plasmodium falciparum, 13.6\% Plasmodium ovale and $1.7 \%$ Plasmodium malaria.
The diagnosis delay between the onset of symptoms and confirmation of the diagnosis of the disease was less than or equal to 2 days in $75 \%$ of cases (Table 3 ). The median of this delay was 2 days $[0.5 ; 2.5]$. This delay can reach 4 days. However, the treatment delay for all cases was zero days; thereafter all cases were taken their treatment the same day of the confirmation of the diagnosis. The parasite identification was done the same day of taking samples in $98.1 \%$ of cases.

\section{Discussion}

The year of 2020, is a principal step for several important health and development goals. This includes efforts to reduce the universal burden of malaria and eliminate the disease where possible as well as efforts to prevent a new introduction of the malaria into the freemalaria countries including the country of Morocco[1].

Our study of the epidemiological profile of the imported malaria into north Morocco is, to our knowledge, the first study carried out in these region since the last indigenous cases notified in 2005 at the region and more specifically in the province of Chefchaouen [4].

Geographically, our results showed that the province of Tangier-Assilah is the most affected province by imported malaria disease. Actually, Tangier-Assilah is the first industrial and commercial pole in the North presenting a continuous increase offoreign exchanges worldwide[11]. The city presents several ports of entry including seaport (Tangier city seaport)or airport (international airport of tangier) makes the province the most exposed to the risk of imported malaria[11]. The second most affected province is Fahs-Anjra with Tangier-Med Port connected to more than 186 ports worldwide, including seaports in African, Asian or South American malaria endemic countries[12]. This presents a potential risk of exposure to the imported malaria for the all-northern region.

An increasing trend of imported malaria cases between 2014 and 2018 at the regional level was observed. This is in accord with the same increasing trend observed in Morocco in the same period [7].

The age of the imported cases in our results $(28.5$ years[23-35]) was lower than the age reported in other studies. The average age of imported malaria cases in the Marrakech-Safi region, Morocco, between 1996 and 2016 was 32 years old[13]. In another study carried out at Agadir University Hospital Centre, the average age of the cases was 38 years old[14].

Men are more affected than women in our study as has been reported in other studies carried out in other regions of Morocco[15, 16]. Also, the men with the age superior to 18 years olds were significantly most affected by malaria disease. These could be explained by the professional activities of the adult male needing travel to the malaria endemic countries. 
Table 2 : The association between gender distribution and age of imported Malaria cases in the north region of Morocco; 2014-2018

\begin{tabular}{lccc}
\hline & \multicolumn{3}{c}{ Gender $($ Frequancy $(\%))$} \\
\cline { 2 - 4 } & Female & Mal & P -value \\
\hline Age range $(\mathbf{n = 5 8 )}$ & $\mathrm{n}=11$ & $\mathrm{n}=47$ & 0,03 \\
$\leq 18$ years & $3(27,2)$ & $5(10,7)$ & \\
$>18$ years & $8(72,7)$ & $42(89,3)$ & \\
Total & $11(19)$ & $47(81)$ & \\
\hline
\end{tabular}

Table 3: Diagnostic, treatment and parasite identification delay of imported malaria cases in the north region of Morocco; 2014-2018

\begin{tabular}{lcc}
\hline & Frequency & Percent $\%$ \\
\hline Diagnostic delay* $(\mathbf{n = 8})$ & & \\
$\leq 2$ days & 6 & 75 \\
$>2$ days & 25 \\
Treatment delay** $(\mathbf{n = 1 3})$ & 13 & 100 \\
0 days & & \\
Parasite identification delay*** $(\mathbf{n}=\mathbf{5 3})$ & 52 & 98,1 \\
0 day & 1 & 1,9 \\
2 days & & \\
\hline
\end{tabular}

* Delay between the date of the onset of symptoms and the date of confirmation of the diagnosis of malaria.

** Delay between the date of the confirmation of the malaria diagnosis and the date of the start of treatment.

*** Delay between the date of the identification of parasite and the date of sampling in the laboratory

The cases with Moroccan nationality are the most reported in our study as well as in the various other studies carried out in Morocco[8].

The travelling of infected people to Guinea and Ivory Coast presented the most important destinations in our study as well as other studies[13, 14, 16, 17]. In addition to African countries [17], there have been also cases imported from Asia and more specifically from the Philippines.

Also, the Plasmodium falciparum parasite is the primary parasite of the imported malaria in Morocco[13, 15,17 ] as well as is our study. The delay from onset of signs to diagnosis (diagnostic delay) of infection in our study ( 2 days) was less than the delay reported in other studies. This delay was 52 days in cases hospitalised in intensive health care in Marrakech [15]. In another study, this delay was between 7.83 and 10.27 days at the Avicenne Military Hospital in Marrakeche [18].

\section{Conclusion and recommendations}

This investigation allows us to describe the epidemiological profile of imported malaria in the north region of Morocco. This is the first study, to our knowledge, carried out in this region since the elimination of malaria in 2010. The region still be exposed to the risk of importing malaria cases from endemic regions in Africa, Asia and others.

To prevent the risk of re-emergence of the disease in our region, malaria prevention and control measures can be at the regional as well as national level. Firstly, detection and rapid clinical care of imported cases of malaria must be carried out. Also, it's needed to support the malaria vector control on the region ports of entry including seaports and airports in accordance with International Health Regulations (IHR), 2005.

On other hand, a last study carried out in 2017 by the Moroccan Ministry of health in cooperation with the Scientific Institute of Morocco including the North region, showed the presence of malaria vector (Anopheles labranchiae) [19]. Therefore, the improvement of vector control must be a priority for the national and regional malaria control program in parallel of the monitoring of vector resistance to pesticides.

Finally, provide the adequate prophylaxis, the advices and information to travellers, whose destination will be an endemic malaria zone, is necessary. This information includes the individual protection measures which have shown their effectiveness against the imported malaria. 


\section{References}

1. World Health Organization, World Malaria Report 2020: 20 years of progress and global challenges. (2020).

2. World Health Organization, Global Technical Strategy for Malaria 2016-2030. (2015).

3. World Health Organization, Adapter les interventions de lutte contre le paludisme dans le contexte de la COVID-19. (2020).

4. Ministère de la Santé, Santé En Chiffre 2005. (2006).

5. M. Rhajaoui, C. Faraj, A. Elaboudi, M. El Aouad, P. R. El Aouad, Analyse du Risque de Maladies Émergentes et Ré-Émergentes au Maroc : Exemple du Paludisme, In Actes de la Session Plénière Solennelle, Académie Hassan II des Sciences et Techniques, 17-19 Février, Rabat, Maroc. (2010).

6. Ministère de la Santé, Santé en Chiffre 2017. (2018).

7. Ministère de la Santé, (DELM), Bulletin d'épidémiologie et de Santé Publique, 56, 76 (2018).

8. Ministère de la Santé, (DELM), Bulletin d'épidémiologie et de Santé Publique, 40, 74 (2017).

9. Ministère de la Santé, Plan Santé 2025 Bilan D'étape Mai 2018-Mai 2019, Rabat, Maroc. (2019).

10. Ministère de la Santé, Projection de la Population par Région, Province et Préfecture, Rabat, Maroc. (2021).

11. Haut-Commissariat Au Plan, Monographie de la Région Tanger-Tétouan-Al Hoceima, Rabat, Maroc. (2020).

12. Rapport Annuel Tanger Med, Https://Www.Tangermed.Ma/WpContent/Uploads/2019/11/Rapport_Annuel_Tan germed Vfr 2018-Web.Pdf. 2018.Accessed In $10 / 06 / 2021$

13. Y. El Guamri, O. Amahmid, K. Zenjari, S. Bouhout, M. A. Mouh, A. A. Melloul, Imported Malaria in the Region of Marrakech-Safi, Morocco, between 1996 and 2016, Bull. Soc. Pathol. Exot, 111, 2 (2018).

14. I. Sellam, Le Paludisme d'importation chez l'adulte, Méd. Mal. Inf, 50, 6 (2020).

15. E. M. El Mezouari, A. Belhadj, M. Ziani, M. Boughanem, And R. Moutaj, Severe imported malaria in adults : a retrospective study of thirteen cases admitted to the Intensive Care Unit in Marrakech, Pan. Afr. Med. J, 25. (2016).

16. B. Charra, M. Sodqi, O. Sandali, H. Nejmi, A. Hachimi, H. Ezzouine, A. Benslama, H. Himmich, S. Motaouakkil, Imported severe malaria in adults : a retrospective study of ten cases admitted to intensive care units in Casablanca, Méd. Mal. Inf, 37, 3 (2007).

17. Y. Larhbali, D. Belghyti, Y. El Guamri, O. Lahlou, K. El Karim, A. Kirami, Z. Khamri, Epidemiology of imported malaria and entomological study of breeding sites of potential risk areas in the province of Khémisset (Maroc), Méd. San. Trop., 24, 4 (2014).

18. M. A. Anouti, Le Paludisme d'importation : expérience de l'hôpital Militaire Avicenne de Marrakech, Phd Thesis,in Faculty of Medicine and Pharmacy of Marrakech..., (2017)

19. C. Faraj, B. Ameur, O. Himmi, M. Sarih, T. Harrak, S. Ouahabi, K. Mustapha, R. Wahabi, A. Maaroufi, Enquête entomologique sur les vecteurs des Arbovirus : Zika, Dengue et Chikungunya au Maroc, 2016-2017, In Bulletin d'épidémiologie et de Santé Publique, Rabat, Maroc. 56. 76 (2018). 